TITLE:

\title{
Enhancement of photoacoustic emission from self-supported plasmonic multilayers
}

$\operatorname{AUTHOR}(S)$ :

Namura, Kyoko; Nakajima, Kaoru; Kimura, Kenji; Suzuki, Motofumi

\section{CITATION:}

Namura, Kyoko ...[et al]. Enhancement of photoacoustic emission from self-supported plasmonic multilayers. Journal of Applied Physics 2014, 116(2): 024310.

\section{ISSUE DATE:}

2014-07-14

URL:

http://hdl.handle.net/2433/192290

\section{RIGHT:}

Copyright 2014 American Institute of Physics. This article may be downloaded for personal use only. Any other use requires prior permission of the author and the American Institute of Physics. 


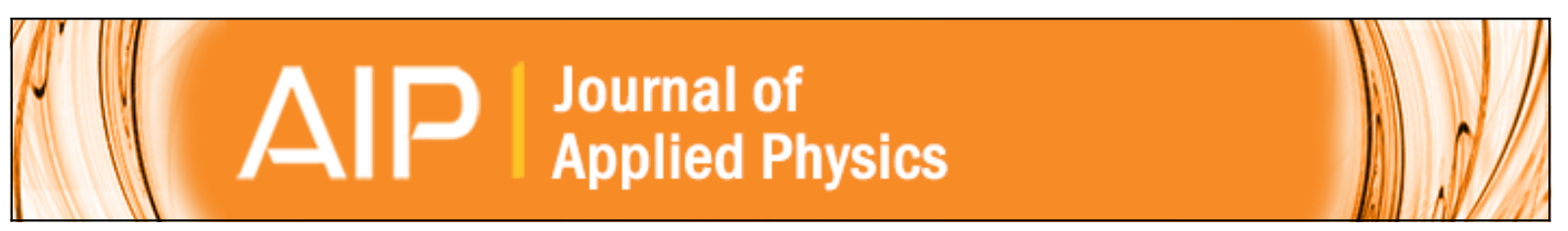

\section{Enhancement of photoacoustic emission from self-supported plasmonic multilayers}

Kyoko Namura, Kaoru Nakajima, Kenji Kimura, and Motofumi Suzuki

Citation: Journal of Applied Physics 116, 024310 (2014); doi: 10.1063/1.4890028

View online: http://dx.doi.org/10.1063/1.4890028

View Table of Contents: http://scitation.aip.org/content/aip/journal/jap/116/2?ver=pdfcov

Published by the AIP Publishing

\section{Articles you may be interested in}

Plasmonic emission enhancement of colloidal quantum dots in the presence of bimetallic nanoparticles

J. Appl. Phys. 115, 134315 (2014); 10.1063/1.4870575

Enhancing the photocurrent and photoluminescence of single crystal monolayer MoS2 with resonant plasmonic nanoshells

Appl. Phys. Lett. 104, 031112 (2014); 10.1063/1.4862745

Enhanced photocurrent in crystalline silicon solar cells by hybrid plasmonic antireflection coatings Appl. Phys. Lett. 101, 261102 (2012); 10.1063/1.4773038

Imaging a spatially confined photoacoustic source defined by a distribution of plasmonic nanoparticles J. Appl. Phys. 111, 094305 (2012); 10.1063/1.4709394

Optical scattering and electric field enhancement from core-shell plasmonic nanostructures

J. Appl. Phys. 110, 103105 (2011); 10.1063/1.3660774

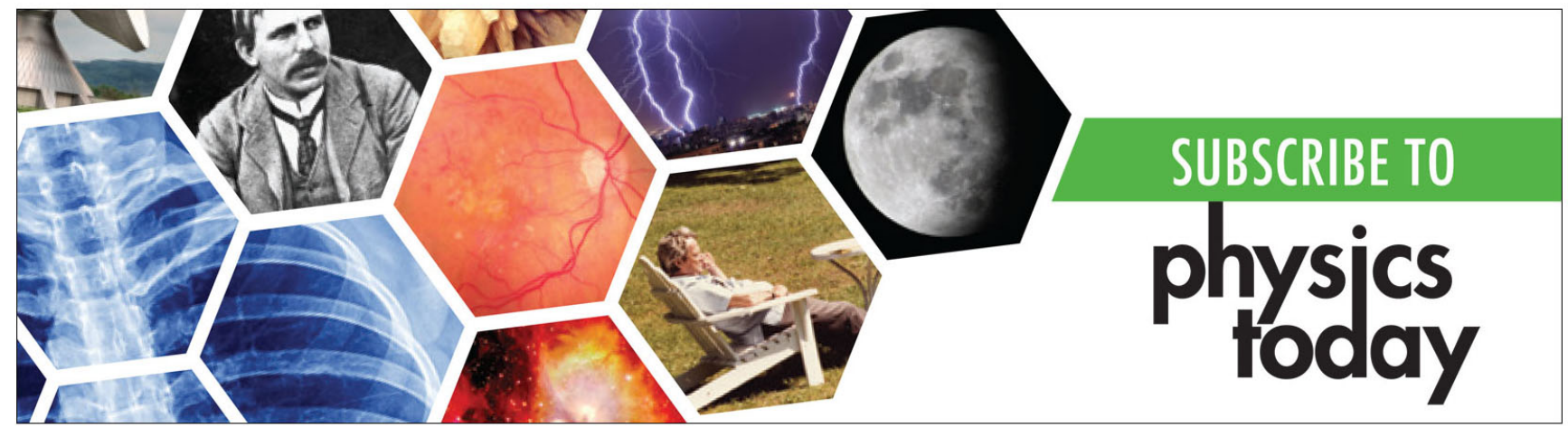




\title{
Enhancement of photoacoustic emission from self-supported plasmonic multilayers
}

\author{
Kyoko Namura, ${ }^{1, a)}$ Kaoru Nakajima, ${ }^{2}$ Kenji Kimura, ${ }^{2}$ and Motofumi Suzuki ${ }^{1}$ \\ ${ }^{1}$ Micro Process Eng. Lab., Department of Micro Engineering, Kyoto University-Katsura, Kyoto 615-8540, \\ Japan \\ ${ }^{2}$ Nanomaterials Eng. Lab., Department of Micro Engineering, Kyoto University-Katsura, Kyoto 615-8540, \\ Japan
}

(Received 6 May 2014; accepted 1 July 2014; published online 11 July 2014)

\begin{abstract}
In this paper, we demonstrate remarkable photoacoustic emission from self-supported Au nanoparticle/dielectric/Ag mirror structures. They were prepared by depositing thin films on both sides of a 50-nm-thick silicon nitride membrane. The photoacoustic emission generated by the self-supported sample is more than 2 orders of magnitude larger than that of a similar structure supported by a Si wafer at a laser modulation frequency of $1 \mathrm{kHz}$. In this frequency region, the thermal diffusion length becomes much greater than the thickness of the multilayers, so that the photoacoustic amplitude is determined mainly by the thermal conductivity of the substrate. Therefore, the large enhancement of the photoacoustic emission can be understood in terms of the reduced thermal conductivity of the substrate. (C) 2014 AIP Publishing LLC. [http://dx.doi.org/10.1063/1.4890028]
\end{abstract}

\section{INTRODUCTION}

Photoacoustic emission from noble metal nanoparticles (NPs) has stimulated much research ${ }^{1}$ and many applications in different fields, including sound-emitting devices, ${ }^{2}$ photoacoustic imaging, 2,3 and manipulation of a fluid or particles by convection flow and thermophoresis. ${ }^{4-6}$ An important challenge for all of these applications is to achieve photoacoustic emission efficiently. The conventional approach to efficient photoacoustic emission is to maximize the optical absorption of the noble metal NPs by manipulating their size and shape. ${ }^{7,8}$ However, the optical absorption of two-dimensional Au NPs arrays has remained only a few tens of percent of the incident light even at the wavelength of the local plasmon resonance. ${ }^{9,10}$

Recently, we successfully demonstrated self-assembly of $\mathrm{Au} \mathrm{NP} /$ porous $\mathrm{SiO}_{2} / \mathrm{SiO}_{2} / \mathrm{Ag}$ mirror structures, namely, local plasmon resonators, ${ }^{11-14}$ using dynamical oblique deposition. The advantages of the local plasmon resonators for photoacoustic emission are the high optical absorption of the Au NP array and the low thermal conductivity of the underlying porous $\mathrm{SiO}_{2}$ layer. First, their multilayered structure acts as an optical cavity and exhibits high optical absorption, $A=0.97$, due to strong interference. This light absorption by the local plasmon resonators is highly localized in their Au NP layer, as expected from transfer matrix and energy balance calculations. ${ }^{13}$ Therefore, they can convert almost all of the incident light energy to heat energy within the two-dimensional $\mathrm{Au}$ $\mathrm{NP}$ array. Second, the presence of the porous $\mathrm{SiO}_{2}$ layer can enhance the heat transfer from the Au NPs to the surrounding fluid because the low thermal conductivity of the porous layer reduces the amount of heat escaping to the substrate. ${ }^{14,15}$ In our previous paper, the photoacoustic emission was enhanced by a factor of 3 by inserting a 340-nm-thick porous $\mathrm{SiO}_{2}$ layer whose thermal conductivity was about 3-4 times lower

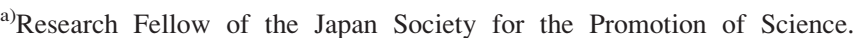
Electronic mail: namura.kyoko.57r@st.kyoto-u.ac.jp
}

than that of a plane $\mathrm{SiO}_{2}$ layer. This result suggests that the reduction in the thermal conductivity of the layer supporting the Au NPs is critical to the enhancement of the photoacoustic emission. If they are held by a thick layer with a significantly low thermal conductivity, we can even expect a dramatic enhancement of the photoacoustic emission.

Here, we focus our attention on the Si substrate, which stretches under the local plasmon resonator, and replace it with air, which has a thermal conductivity that is more than 3 orders of magnitude lower than that of $\mathrm{Si}^{16}$ In this study, we propose a method of fabricating self-supported local plasmon resonators and show their remarkable photoacoustic emission.

\section{EXPERIMENTS}

In our previous study, ${ }^{13}$ we demonstrated the fabrication of local plasmon resonators supported by a $\mathrm{Si}$ wafer, as depicted in Fig. 1(a). Here, we propose a method of fabricating self-supported $\mathrm{Au} \mathrm{NP/shape} \mathrm{control} \mathrm{layer} \mathrm{(SCL)/phase}$ control layer (PCL) $/ \mathrm{Si}_{3} \mathrm{~N}_{4} / \mathrm{Ag}$ mirror structures (Fig. 1(b)), i.e., self-supported local plasmon resonators, by dynamical oblique deposition. We used a commercial silicon nitride membrane ( $50 \mathrm{~nm}$ thick, $1 \mathrm{~mm}$ square) and deposited thin (a)

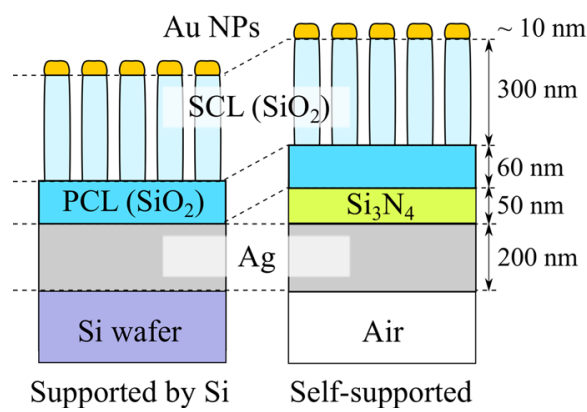

(c)

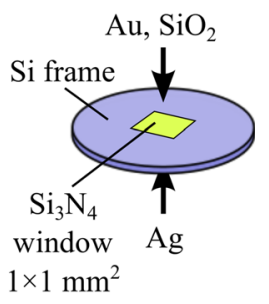

$1 \times 1 \mathrm{~mm}^{2}$
FIG. 1. Schematic drawing of local plasmon resonators 
films on both sides of it (Fig. 1(c)). First, a smooth Ag layer $200 \mathrm{~nm}$ in thickness was deposited on one side of the membrane. Then, on the other side of the membrane, a PCL of 60-nm-thick $\mathrm{SiO}_{2}$ was deposited from the normal direction to the surface. On the PCL, an SCL of $\mathrm{SiO}_{2}$ up to $300 \mathrm{~nm}$ thick was prepared. During SCL deposition, the deposition angle measured from the surface normal was fixed at $78.6^{\circ}$, and the substrate was rotated continuously and rapidly. As a result, $\mathrm{SiO}_{2}$ nanopillars on the PCL were obtained. Finally, an $\mathrm{Au}$ NP layer $8.6 \mathrm{~nm}$ in thickness was deposited on the SCL at a deposition angle of $83.4^{\circ}$. We also fabricated local plasmon resonators supported by a Si wafer (Fig. 1(a)) using the fabrication method reported in our previous paper. ${ }^{13}$

Optical reflection measurements at an angle of incidence of $2^{\circ}$ were conducted using a single-beam spectrophotometer in the wavelength range of $300 \mathrm{~nm}<\lambda<1700 \mathrm{~nm}$. Because the optical transmittance and scattering of our samples are negligibly small, their optical absorption $A$ was evaluated as $A=1-R$, where $R$ is the optical reflectance.

The photoacoustic measurements ${ }^{14}$ were performed using a photoacoustic cell $(10.0 \mathrm{~mm}$ in diameter and $3.5 \mathrm{~mm}$ in height, MTEC Model 300) on the self-supported local plasmon resonators and, for comparison, on those supported by a $\mathrm{Si}$ wafer. We loaded the sample, which has an area of $1 \mathrm{~mm} \times 1 \mathrm{~mm}$, onto the photoacoustic cell filled with air. Then, the sample was irradiated by a laser (wavelength $785 \mathrm{~nm}$ ), the intensity of which was modulated sinusoidally. The angle of incidence of the laser was perpendicular to the sample surface. The photoacoustic signal detected by a microphone was measured with a lock-in amplifier and recorded as a function of the laser modulation frequency over a range of $1 \mathrm{kHz}-100 \mathrm{kHz}$.

\section{RESULTS AND DISCUSSION}

Figure 2 shows typical cross-sectional scanning electron microscopy (SEM) images of (a) the self-supported sample and (b) the sample supported by the Si wafer. The selfsupported sample (Fig. 2(a)) is held by the smooth 50-nmthick $\mathrm{Si}_{3} \mathrm{~N}_{4}$ membrane, which can be found between the $\mathrm{Ag}$ and the PCL. Because the SEM image of the self-supported sample was taken from a slightly tilted angle with respect to the sample surface, the lower surface of the Ag layer, which appears to be glowing white, is visible. These SEM observations suggest that the self-supported local plasmon resonators with the Au NP/SCL/dielectric spacer/Ag mirror layer structure depicted in Fig. 1(b) were successfully fabricated as intended by dynamical oblique deposition.
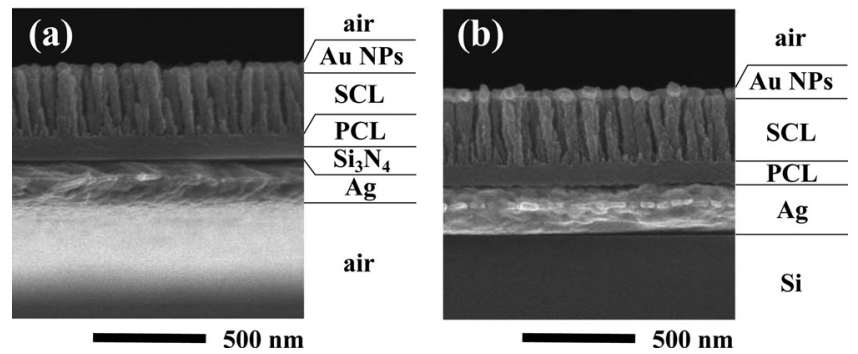

FIG. 2. Typical cross-sectional SEM images of (a) self-supported local plasmon resonator and (b) resonator supported by air.

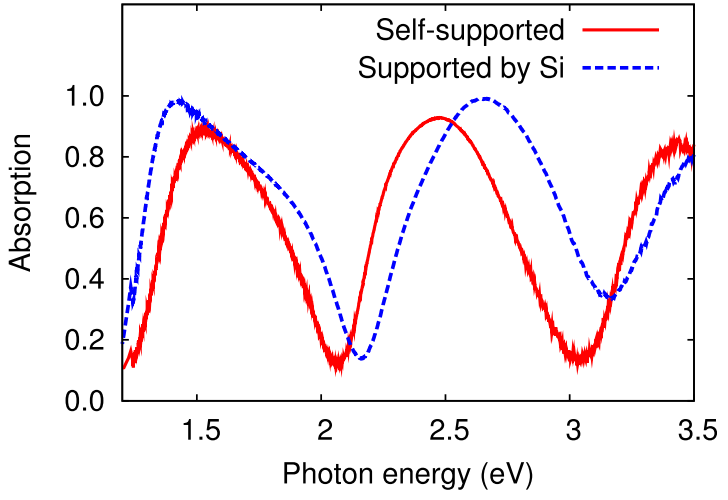

FIG. 3. Optical absorption spectra of self-supported local plasmon resonator (red solid line) and resonator supported by Si wafer (blue dashed line).

Figure 3 shows typical optical absorption spectra of the self-supported sample (red solid line) and the sample supported by a Si wafer (blue dashed line). The sample supported by a $\mathrm{Si}$ wafer (blue dashed line) shows a periodic change in its optical absorption as a function of the photon energy due to interference, which is a typical spectral feature of local plasmon resonators. ${ }^{11,13}$ Their optical properties can be understood in terms of multiple homogeneous layers, at least around the near-infrared or longer-wavelength region, because the dimensions of the nanostructures in the layers are much smaller than the wavelength. Although the self-supported sample has an additional $\mathrm{Si}_{3} \mathrm{~N}_{4}$ layer in the dielectric layers of the local plasmon resonator, it shows spectral features similar to those of the sample supported by a $\mathrm{Si}$ wafer. This result suggests that the presence of the $\mathrm{Si}_{3} \mathrm{~N}_{4}$ layer affects only the phase of the interference, and optically equivalent structures were successfully fabricated on both $\mathrm{Si}$ and air substrates. Among the fabricated samples, the two depicted in Fig. 3 showed almost the same optical absorption at a wavelength of $785 \mathrm{~nm}$ $(1.58 \mathrm{eV})$. These samples are suitable for investigating the contribution of the thermal properties of the substrate to the photoacoustic emission from the local plasmon resonators. Therefore, we performed photoacoustic measurements on those samples using a 785-nm-wavelength laser and compared their photoacoustic emission.

Figure 4 shows the photoacoustic spectra of the selfsupported sample (solid line) and the sample supported by a

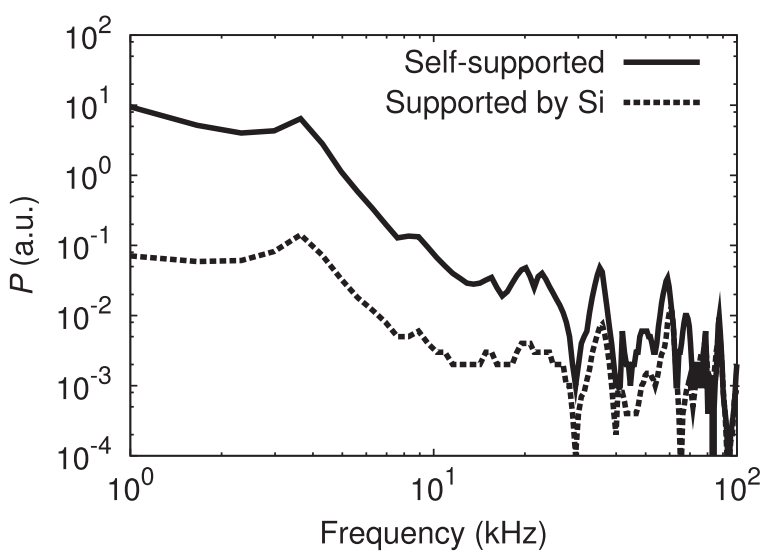

FIG. 4. Photoacoustic spectra of self-supported local plasmon resonator (solid line) and resonator supported by Si wafer (dashed line). 
Si wafer (dashed line) as a function of the laser modulation frequency. These spectra have many distinct peaks at identical positions. The peaks originate in the acoustic resonance in the photoacoustic cell. Apart from these resonance peaks, we focused our attention on the relative amplitudes of the obtained spectra, which qualitatively describe the surface temperature modulation of the samples. ${ }^{17}$ Although the selfsupported sample and the sample supported by a Si wafer exhibit almost the same optical absorption, the photoacoustic amplitude $P$ of the self-supported sample is more than 2 orders of magnitude larger than that of the sample supported by a $\mathrm{Si}$ wafer at a laser modulation frequency of $1 \mathrm{kHz}$. This result suggests that the photoacoustic amplitude of the Au NP array is significantly improved by replacing the Si substrate with air, which has a thermal conductivity about 3 orders of magnitude lower than that of a Si wafer. ${ }^{16}$

To quantitatively compare the photoacoustic amplitude of the self-supported sample, $P_{\text {air }}$, and that of the sample supported by a Si wafer, $P_{s i}$, we plotted $P_{\text {air }} / P_{s i}$ as a function of the laser modulation frequency (Fig. 5, black dots). The value of $P_{\text {air }} / P_{s i}$ increased rapidly with decreasing laser modulation frequency. This frequency characteristic of $P_{\text {air }} / P_{s i}$ is expected to be understood by heat transfer analysis because the photoacoustic amplitude is known to arise from the periodic temperature change of the sample surface. ${ }^{17}$ Therefore, we evaluated $P_{\text {air }} / P_{s i}$ using a one-dimensional heat transfer model, the procedure for which is essentially the same as that in our previous work. ${ }^{14}$

According to the derivation of Rosencwaig and Gersho, ${ }^{17}$ the relationship between the sample surface temperature and the photoacoustic amplitude can be written as

$$
P=F \frac{\left|U^{a c}\right|}{\mid U^{d c \mid}},
$$

where $U^{a c}$ and $U^{d c}$ are the AC and DC components of the sample surface temperature, respectively, and $\mathrm{F}$ is a constant that is determined by the shape of the photoacoustic cell and the physical properties of the surrounding gas. Because the laser irradiation power is not very strong, and $U^{d c}$ is estimated to be about room temperature, ${ }^{14}$ we can assume $P_{\text {air }} / P_{s i}=\left|U_{\text {air }}^{a c}\right| /\left|U_{s i}^{a c}\right|$, where the subscripts "air" and "si"

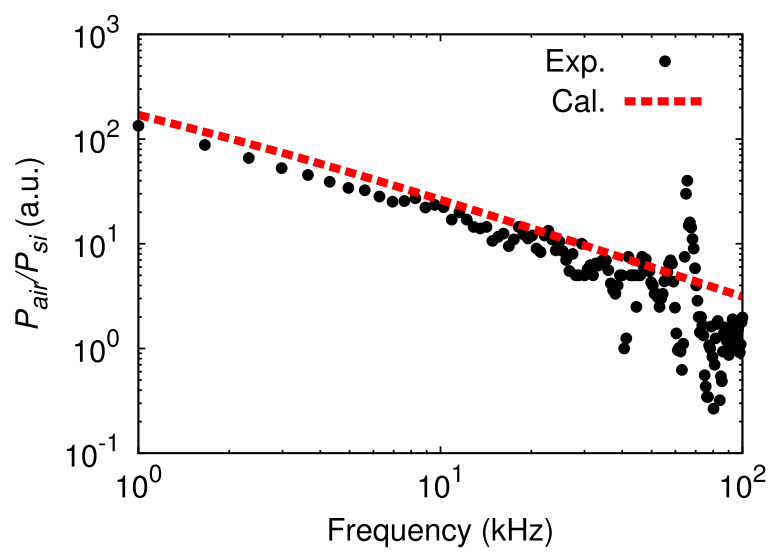

FIG. 5. Ratio of the photoacoustic amplitudes of self-supported sample and sample supported by $\mathrm{Si}$ wafer, $P_{\text {air }} / P_{s i}$, as a function of laser modulation frequency (black dots). Red dashed line shows the calculation result. represent the self-supported sample, which has an air substrate, and the sample supported by a Si wafer, respectively. The values of $\left|U_{s i}^{a c}\right|$ and $\left|U_{\text {air }}^{a c}\right|$ can be estimated by numerical calculation using a one-dimensional thermal diffusion equation if the thickness, thermal conductivity, and thermal diffusivity of each layer are defined.

Here, we assume that the systems of the self-supported local plasmon resonator and the resonator supported by a $\mathrm{Si}$ wafer in the photoacoustic cell are composed of air $(500 \mu \mathrm{m}) / \mathrm{SCL} \quad(300 \mathrm{~nm}) / \mathrm{PCL} \quad(60 \mathrm{~nm}) / \mathrm{Si}_{3} \mathrm{~N}_{4} \quad(50 \mathrm{~nm}) /$ air $(500 \mu \mathrm{m})$ and air $(500 \mu \mathrm{m}) / \mathrm{SCL}(300 \mathrm{~nm}) / \mathrm{PCL}(60 \mathrm{~nm}) / \mathrm{Si}$ $(500 \mu \mathrm{m})$, respectively. To eliminate the effect of the cell walls on the calculations, the air layer and Si layer are taken to be much thicker than their thermal diffusion length at $1 \mathrm{kHz}$, which is expressed by $\lambda=\sqrt{a / \pi f}$, where $a$ is the thermal diffusivity $\left(\mathrm{m}^{2} / \mathrm{s}\right)$, and $f$ is the laser modulation frequency $\left(s^{-1}\right)$. The thermal conductivity and diffusivity of the layers are listed in the Table I. For the PCL $\left(\mathrm{SiO}_{2}\right)$ and $\mathrm{Si}_{3} \mathrm{~N}_{4}$ layer, we used the values of those thin films from the literature. ${ }^{18,19}$ The $\mathrm{Si}$ and air layers were considered as bulk layers, the thermal properties of which are given in the literature. ${ }^{16}$ In addition, the thermal properties of the SCL, which can be treated as a homogeneous layer with a thermal conductivity 4 times lowers than that of the PCL, were described in our previous paper. ${ }^{14}$ Although the actual local plasmon resonators have an $\mathrm{Ag}$ mirror layer $(200 \mathrm{~nm})$ on the Si wafer, its thickness is significantly smaller than the thermal diffusion length of $\mathrm{Ag}$ and is negligible because of the uniformity of the temperature inside the layer. Furthermore, we assume that the Au NP layer at the top of the sample is a heat source whose thickness is negligibly small because the thickness of the Au NP layer is significantly smaller than those of the other layers, and the incident light is absorbed and converted into heat by the NP layer. ${ }^{13,14}$ We define the sample surface to be at $x=0$; hence, the heat applied by photothermal conversion is assumed to be

$$
q(x, t)=A Q_{0}(1+\cos 2 \pi f t) \delta(x),
$$

where $t$ is the time (s), and $Q_{0}$ is the power density of the incident light $\left(\mathrm{W} / \mathrm{m}^{2}\right)$. Because, as described earlier, $U_{d c}$ for the sample surface is estimated to be about room temperature, and also the amplitude of $U_{a c}$ is sufficiently small, we can assume that air convection in the cell, which is caused by the temperature gradient, is negligibly small. Therefore, the heat diffusion equation for each layer is written as

$$
\frac{\partial u(x, t)}{\partial t}=a \frac{\partial^{2} u(x, t)}{\partial x^{2}},
$$

where $u$ is the temperature $(\mathrm{K})$. Equation (3) is solved under the boundary conditions that the temperature and heat flux

TABLE I. Thermal conductivity $\kappa$ and thermal diffusivity $a$ of each layer.

\begin{tabular}{lccccc}
\hline \hline Materials & \multirow{2}{*}{$\mathrm{Air}^{16}$} & $\mathrm{PCL}^{18}$ & $\mathrm{SCL}^{14}$ & $\mathrm{Si}_{3} \mathrm{~N}_{4}{ }^{19}$ & $\mathrm{Si}^{16}$ \\
\hline$\kappa(\mathrm{W} / \mathrm{m} \mathrm{K})$ & $2.4 \times 10^{-2}$ & $8.0 \times 10^{-1}$ & $2.0 \times 10^{-1}$ & $2.7 \times 10^{0}$ & $1.5 \times 10^{2}$ \\
$a\left(\mathrm{~m}^{2} / \mathrm{s}\right)$ & $1.85 \times 10^{-5}$ & $3.70 \times 10^{-7}$ & $3.70 \times 10^{-7}$ & $1.24 \times 10^{-6}$ & $8.95 \times 10^{-5}$ \\
\hline \hline
\end{tabular}


are continuous at interfaces. In addition, we assume that the temperature at the cell walls, which are far from the heat source, is room temperature.

The red dashed line in Fig. 5 is the calculation result, which agrees well with the experimental result, shown by black dots. This result suggests that reducing the thermal conductivity of the substrate by replacing the $\mathrm{Si}$ wafer with air contributes to the enhancement of the photoacoustic emission. At a laser modulation frequency of $1 \mathrm{kHz}$, the thermal diffusion lengths of the PCL $(\lambda \simeq 10.8 \mu \mathrm{m}), \mathrm{SCL}$ $(\lambda \simeq 10.8 \mu \mathrm{m})$, and $\mathrm{Si}_{3} \mathrm{~N}_{4}(\lambda \simeq 19.9 \mu \mathrm{m})$ are much greater than the thickness of the local plasmon resonators composed of those materials. Consequently, the thermal properties of the substrate, i.e., Si or air, under the multilayered thin film dominate the photoacoustic amplitude, and the reduction of the thermal conductivity has a pronounced effect on it.

\section{CONCLUSION}

We demonstrated the fabrication of self-supported local plasmon resonators and their photoacoustic emission. Using silicon nitride membranes, self-supported Au NP/PCL/SCL/ $\mathrm{Si}_{3} \mathrm{~N}_{4} / \mathrm{Ag}$ structures were successfully fabricated. At a laser modulation frequency of $1 \mathrm{kHz}$, the photoacoustic amplitude of the self-supported sample is more than 2 orders of magnitude larger than that of the sample supported by a Si wafer. This large enhancement of the photoacoustic emission is due to a reduction in the thermal conductivity of the substrate caused by replacing the $\mathrm{Si}$ wafer with air, which was verified using a one-dimensional heat transfer model. In addition, the thermal diffusion length becomes much longer than the thickness of the local plasmon resonators at a laser modulation frequency of $1 \mathrm{kHz}$, so the photoacoustic emission significantly reflects the change in the thermal properties of the substrate. Consequently, the self-supported local plasmon resonators are suitable for use as sound-emitting devices in the audible frequency range.

\section{ACKNOWLEDGMENTS}

We are grateful to Sadamu Kinoshita from Kyoto University for assisting us with the SEM observations. This work was supported by JSPS KAKENHI Grant Nos. 25286037 and $24 \cdot 2362$.

${ }^{1}$ V. P. Zharov, Nat. Photonics 5(2), 110-116 (2011).

${ }^{2}$ Y. Hou, J.-S. Kim, M. O’Donnel, and L. J. Guo, Appl. Phys. Lett. 89, 093901 (2006).

${ }^{3}$ Y. S. Chen, W. Frey, S. Kim, P. Kruizinga, K. Homan, and S. Emelianov, Nano Lett. 11(2), 348-354 (2011).

${ }^{4}$ V. P. Zharov, T. V. Malinsky, and R. C. Kurten, J. Phys. D: Appl. Phys. 38, 2662-2674 (2005).

${ }^{5}$ D. Braun and A. Libchaber, Phys. Rev. Lett. 89(18), 188103 (2002).

${ }^{6}$ L. H. Thamdrup, N. B. Larsen, and A. Kristensen, Nano Lett. 10(3), 826-832 (2010).

${ }^{7}$ W. Gotschy, K. Vonmetz, A. Leitner, and F. R. Aussenegg, Opt. Lett. 21(15), 1099-1101 (1996).

${ }^{8}$ W. Hasan, C. L. Stender, M. H. Lee, C. L. Nehl, J. Lee, and T. W. Odom, Nano Lett. 9(4), 1555-1558 (2009).

${ }^{9}$ B. Auguié and W. L. Barnes, Phys. Rev. Lett. 101, 143902 (2008).

${ }^{10}$ J. Parsons, E. Hendry, C. P. Burrows, B. Auguié, J. R. Sambles, and W. L. Barnes, Phys. Rev. B 79, 073412 (2009).

${ }^{11}$ M. Suzuki, Y. Imai, H. Tokunaga, K. Nakajima, K. Kimura, T. Fukuoka, and Y. Mori, J. Nanophotonics 3, 031502 (2009).

${ }^{12}$ K. Namura, M. Suzuki, K. Nakajima, and K. Kimura, Opt. Lett. 36, 3533-3535 (2011).

${ }^{13}$ K. Namura, M. Suzuki, K. Nakajima, and K. Kimura, J. Appl. Phys. 114, 074308 (2013).

${ }^{14}$ K. Namura, M. Suzuki, K. Nakajima, and K. Kimura, Opt. Express 21(7), 8689-8700 (2013).

${ }^{15}$ H. Shinoda, T. Nakajima, K. Ueno, and N. Koshida, Nature 400, 853-855 (1999).

${ }^{16}$ National Astronomical Observatory of Japan, Chronological Science Tables (Maruzen, Tokyo, 1993).

${ }^{17}$ A. Rosencwaig and A. Gersho, J. Appl. Phys. 47(1), 64-69 (1976).

${ }^{18}$ S.-M. Lee and D. G. Cahill, J. Appl. Phys. 81(6), 2590-2595 (1997).

${ }^{19}$ M. T. Alam, M. P. Manoharan, M. A. Haque, C. Muratore, and A. Voevodin, J. Micromech. Microeng. 22, 045001 (2012). 